\title{
Methotrexate and anti-tumor necrosis factor treatment improves endothelial function in patients with inflammatory arthritis
}

\author{
Gia Deyab ${ }^{1 *}$ (D) Ingrid Hokstad ${ }^{2}$, Jon Elling Whist ${ }^{1,11}$, Milada Cvancarova Smastuen ${ }^{3}$, Stefan Agewall ${ }^{4,5}$, \\ Torstein Lyberg ${ }^{6}$, Nicoletta Ronda ${ }^{7}$, Knut Mikkelsen², Gunnbjorg Hjeltnes ${ }^{8}$ and Ivana Hollan 2,9,10,11
}

\begin{abstract}
Background: Inflammatory arthritis (IA), including rheumatoid arthritis (RA), ankylosing spondylitis (AS) and psoriatic arthritis (PSA), leads to increased cardiovascular disease occurrence probably due to atherosclerosis. One of the first stages in atherogenesis is endothelial dysfunction (ED). Therefore, we aimed to compare endothelial function (EF) in patients with IA, and to examine the effects of methotrexate (MTX) monotherapy and antitumor necrosis factor (anti-TNF) treatment with or without MTX comedication (anti-TNF \pm MTX) on EF.

Methods: From the PSARA observational study, all patients with RA $(n=64)$, PSA $(n=29)$, and AS $(n=20)$ were evaluated for EF. In patients with ED at baseline $(n=40)$, we evaluated changes in the Reactive Hyperemic Index $(\mathrm{RHI})$ after 6 weeks and 6 months of antirheumatic therapy.

Results: In IA patients with ED, RHI significantly improved after 6 weeks $(p<0.001)$ and 6 months $(p<0.001)$ of treatment, independent of changes in disease activity parameters. After 6 months, RHI had improved more in the MTX group than in the anti-TNF \pm MTX group, and the difference remained statistically significant after adjustments for potential confounders. Among patients with active RA, AS, and PsA, those with AS appeared to have the worst endothelial function, although they were the youngest.
\end{abstract}

Conclusion: Treatment with MTX and anti-TNF \pm MTX was associated with a relatively fast improvement of EF in IA patients with ED, independent of change in disease activity. Therefore, modes of action other than the antiinflammatory effect may contribute to the EF improvement. After 6 months, the EF improvement was more pronounced in the MTX group than in the anti-TNF \pm MTX group.

Trial registration: Clinicaltrials, NCT00902005. Registered on 13 May 2009.

Keywords: Inflammatory arthritis, Methotrexate, Anti-tumor necrosis factor, Rheumatic arthritis, Spondyloarthritis

\section{Background}

Inflammatory arthritis (IA), including rheumatoid arthritis (RA), ankylosing spondylitis (AS), and psoriatic arthritis (PsA), has increased cardiovascular (CV) morbidity and mortality, probably due to cardiovascular disease (CVD) caused by atherosclerosis [1-5]. The first step in the development of atherosclerosis is endothelial dysfunction

\footnotetext{
* Correspondence: gyyadeyab@gmail.com

'Department of Medical Biochemistry, Innlandet Hospital Trust, Hamar, Norway

Full list of author information is available at the end of the article
}

(ED) which is initially a reversible process [6]. Thus, improving endothelial function (EF) might be of great importance in preventing atherosclerosis. The endothelium has several vital homeostatic functions, including regulation of vascular tone and growth, thrombogenesis and thrombolysis, and interactions between platelets and leukocytes and the vessel wall. The endothelium secretes vasorelaxing (e.g., nitric oxide) and vasoconstricting (e.g., endothelin-1) substances in response to mechanical stress $[6,7]$. ED is characterized by impaired ability of the artery to dilate in response to physical and chemical stimuli $[8,9]$. 
Assessment of $\mathrm{EF}$ has been used to estimate the $\mathrm{CV}$ risk in IA patients $[10,11]$.

Clinical studies indicate that antirheumatic treatment, including methotrexate (MTX) and antitumor necrosis factor (anti-TNF) treatment, not only ameliorates disease activity but also reduces $\mathrm{CV}$ morbidity and mortality in RA patients $[12,13]$. There is also evidence that antiTNF treatment improves EF in RA, and reduces arterial stiffness and intima-media thickness in patients with RA, PsA, and AS [14, 15].

However, information on the effect of antirheumatic drugs on EF in AS and PsA patients is still limited. Therefore, the aim of this study was to compare EF in RA, AS, and PsA patients, and to examine the effect of antirheumatic treatment (MTX and/or anti-TNF) on EF in these patient groups.

\section{Methods}

\section{Patients}

We examined patients from the PSoriatic arthritis, Ankylosing spondylitis, Rheumatoid Arthritis (PSARA) study who completed 6 months of follow-up and in whom EF was measured $(n=113)$. Of the 114 patients who completed the study, one PsA patient was excluded because she was not able to adhere to the requirements of the EF measurement (smoked and did not sit still).

All patients in PSARA, an observational study, had been included at the Lillehammer Hospital for Rheumatic Diseases as described elsewhere [16]. Briefly, the inclusion criteria were: males and females with an age range 18-80 years; and PsA according to the Moll and Wright 1973 criteria [17], AS according to the modified New York diagnostic criteria for AS [18], or RA according to the ACR 1987 criteria [19], and clinical indication for starting with either MTX monotherapy or anti-TNF treatment with or without MTX comedication (anti-TNF \pm MTX).

Exclusion criteria included lack of cooperability, any contraindication for MTX and anti-TNF, any significant infection (including subclinical tuberculosis), pregnancy or breastfeeding, congestive heart failure, use of systemic glucocorticoids $>10 \mathrm{mg} /$ day during the last 2 weeks or anti-TNF during the last 4 weeks before the inclusion, and any chronic inflammatory disease other than RA, AS, or PsA.

All patients were Caucasian and were examined at baseline and after 6 weeks and 6 months of treatment.

\section{Treatment}

Patients were either treated with MTX monotherapy or with anti-TNF \pm MTX. The type and doses of antirheumatic treatment were decided by rheumatologists not involved in the study upon clinical judgment and in accordance with Norwegian guidelines. Doses were as follows: etanercept $50 \mathrm{mg}$ subcutaneous injection once a week; infliximab 3-5 $\mathrm{mg} / \mathrm{kg}$ intravenous injection at baseline, then following standard dosing regimen; adalimumab $40 \mathrm{mg}$ subcutaneous injection every other week; MTX 15-25 mg orally once a week.

Norwegian guidelines consider MTX as a first-line antirheumatic treatment in patients with chronic peripheral arthritis, in particular RA [20]. Due to limited effects of conventional disease-modifying antirheumatic drugs (DMARDs) in axial spondyloarthritis (SpA), including AS and PsA, TNF inhibition is used in SpA patients with axial disease who do not sufficiently respond to nonsteroidal anti-inflammatory drugs (NSAIDs) [21, 22]. Throughout the study period, patients using glucocorticoids were kept on a steady dose (10 $\mathrm{mg}$ or less per day).

\section{Clinical and laboratory tests}

Data collection included demographic data, medical history, physical findings, lifestyle information and medication.

EF was examined, and blood samples were drawn after fasting for $8 \mathrm{~h}$ (including nonallowance of smoking), and hospital routine blood tests were consecutively performed.

EF was evaluated using a reactive hyperemia peripheral arterial tonometry (RH-PAT) examination which evaluates the overall health of the endothelium by measurment of finger arterial pulsatile volume changes as described previously [23]. The Reactive Hyperemic Index (RHI) was calculated as the ratio between the magnitude of the average postobstructive pulse wave amplitude (PWA) and the average of baseline PWA (preocclusion). ED was defined as $\mathrm{RHI} \leq 1.67$ as recommended by the manufacturer and in accordance with findings from a population at risk for ischemic heart disease [23]. RHI results for a subgroup of our RA sample have been published previously [24].

We evaluated improvement in RHI only in patients with ED, as a significant improvement in RHI could not be expected in patients with normal EF.

\section{Statistics}

For comparisons of continuous independent variables between and within the examined groups, nonparametric tests (Mann-Whitney $U$ test and Wilcoxon sign test) were applied, since the continuous variables of interest were not normally distributed (according to normality plots). For comparison of categorical data between the study groups, the Chi-square test was used. Linear regression analyses were used to assess associations between RHI change modeled as the dependent variable (baseline and 6 months) and selected disease activity markers. Age, gender, rheumatic diagnosis, and variables that showed a significant association with the dependent variable in simple regression analyses were included in multiple linear regression models.

$P$ values $\leq 0.05$ were considered statistically significant, and all statistical tests were two-sided. Our analyses 
were considered exploratory so no correction for multiple testing was performed.

All analyses were performed using IBM SPSS statistics, version 23.

\section{Results}

\section{Baseline patient characteristics}

Baseline clinical and cardiovascular characteristics of all patients who completed the 6 months of follow-up are described in Tables 1 and 2.

The anti-TNF \pm MTX and MTX groups had similar characteristics except for a significantly shorter rheumatic disease duration and higher Physicians' Global Assessment (PGA) score in the MTX group ( $p=0.043$ and $p=0.002$, respectively). The proportion of patients with ED was similar in both treatment groups.

Although patients with AS were the youngest (statistically significantly younger than the RA group), they had higher frequency of $\mathrm{ED}$, angina pectoris, myocardial infarction, and use of some cardiovascular drugs (beta blockers, statins and warfarin) compared to the RA and PsA groups (these differences did not reach the level of statistical significance).

The AS group had the lowest median RHI value, which was significantly different from the PsA group $(p=0.040$; Fig. 1). The proportion of women was highest in the RA group and lowest in the AS group (Table 1).

When evaluating only patients with ED, there were no statistically significant differences in RHI baseline values between any of the three diagnostic groups.

\section{RHI improvement in patients with ED}

In the total IA group with ED $(n=40)$, RHI significantly improved from baseline to 6 weeks $\left(\mathrm{RHI}_{6 \text { weeks }}=1.86, p\right.$ $<0.001)$, and from baseline to 6 months $\left(\mathrm{RHI}_{6 \text { months }}=\right.$ 1.80, $p<0.001$ ) (Fig. 1). RHI baseline levels are described in Table 2.

The RHI improvement was most pronounced at 6 weeks. At 6 months, the RHI median level slightly, but statistically nonsignificantly, decreased again (Fig. 1).

In analyses of all three diagnostic groups with ED, only RA patients showed statistically significant RHI improvement from baseline to 6 weeks (RHI at 6 weeks = 1.96, $p<0.001$ ) and baseline to 6 months (RHI at 6 months $=1.86, p=0.001$; Fig. 1). The PsA group showed RHI improvement at both visits (RHI at 6 weeks = 1.67 and RHI at 6 months $=1.80$ ). In the AS group the RHI levels slightly decreased from baseline to 6 weeks (RHI at 6 weeks $=1.50)$. However, after 6 months of treatment, the RHI levels increased again (RHI at 6 months $=1.68$ ). None of the RHI changes in the PsA and AS groups reached statistical significance.

\section{Effect of MTX and anti-TNF \pm MTX on RHI in patients with ED}

In both treatment groups, RHI significantly improved at both follow-up visits compared to baseline (MTX: baseline to 6 weeks $p=0.002$, baseline to 6 months $p=0.001$; anti-TNF \pm MTX: baseline to 6 weeks $p=0.004$, baseline to 6 months $p=0.024$ ). After 6 months of treatment, RHI values in the MTX group continued to increase compared to 6 weeks. However, in the anti-TNF \pm MTX group RHI values at 6 months were lower than at 6 weeks, resulting in a statistically significant difference in RHI values between the two groups at 6 months (Fig. 2).

Within the RA and PsA groups there were no significant differences in RHI between patients treated with MTX and anti-TNF \pm MTX.

\section{Linear regression analysis}

Our data did not reveal any statistically significant associations between RHI and inflammatory markers, including C-reactive protein (CRP), white blood cell (WBC) count, erythrocyte sedimentation rate (ESR), pentraxin (PTX)3, Modified Health Assessment Questionnaire (MHAQ), PGA, or Patients' Global Assessment Score of Disease Activity (PtGA), at baseline (data not shown).

In simple regression analyses, only female gender and rheumatic disease duration were significantly related to RHI change from baseline to 6 months, while age, IA diagnosis, changes in markers of IA activity and severity (CRP, WBC count, ESR, PTX3, MHAQ, PGA, and PtGA) (Table 3), traditional CV risk factors (smoking, hypertension, diabetes, body mass index, established CVD (history of previous myocardial infarctions and presence of angina) and medications (statins, angiotensin converting enzyme inhibitors, and calcium antagonists); data not shown) were not.

Female gender was related to a greater improvement in RHI compared to male gender, and the association remained statistically significant in several multiple regression models including models adjusted for age, rheumatic disease duration, and IA diagnosis. Rheumatic disease duration was negatively related to RHI change from baseline to 6 months and it stayed statistically significant in several multiple regression models (adjusted for age, gender, and IA diagnosis and age, gender, and treatment).

The difference in RHI change from baseline to 6 months between the MTX group and the anti-TNF \pm MTX group remained statistically significant after adjustments for age, female gender, rheumatic disease duration, and IA diagnosis (Table 3).

\section{Corrections for baseline RHI values}

In analyses adjusted for baseline RHI values, MTX was associated with a greater improvement in RHI than anti$\mathrm{TNF} \pm$ MTX after 6 months in patients with $\mathrm{ED}(p=0.007)$. 
Table 1 Baseline clinical characteristics for all patients

\begin{tabular}{|c|c|c|c|c|c|}
\hline & $\begin{array}{l}\text { RA } \\
(n=64)\end{array}$ & $\begin{array}{l}\text { PsA } \\
(n=29)\end{array}$ & $\begin{array}{l}\text { AS } \\
(n=20)\end{array}$ & $\begin{array}{l}\text { MTX } \\
(n=49)\end{array}$ & $\begin{array}{l}\text { anti-TNF } \pm \text { MTX } \\
(n=64)\end{array}$ \\
\hline Age (years) & $57(28-79)$ & $50(23-78)^{7}$ & $49(30-72)^{\phi}$ & $56(28-79)$ & $55(23-75)$ \\
\hline Women, $n(\%)$ & $47(73)$ & $12(41)^{*}$ & $4(20)^{\phi \epsilon}$ & $30(61)$ & $33(52)$ \\
\hline Rheumatic disease duration (years) & $2(0-30)$ & $3(0-37)$ & $3(0-40)$ & $0.10(0-25)$ & $3.7(0-40)^{*}$ \\
\hline \multicolumn{6}{|l|}{ Disease activity } \\
\hline $\mathrm{CRP}(\mathrm{mg} / \mathrm{L})$ & $8(1-78)$ & $5(1-99)$ & $10(1-157)$ & $8(1-99)$ & $6.5(1-157)$ \\
\hline $\operatorname{ESR}(\mathrm{mm} / \mathrm{h})$ & $18.5(1-81)$ & $7(2-48)^{¥}$ & $9.5(2-87)^{\phi}$ & $14(1-81)$ & $13(2-87)$ \\
\hline Anti-CCP, n (\%) & $39(61)$ & - & - & $17(35)$ & $22(34)$ \\
\hline Rheumatoid factor IgA, $n$ (\%) & $32(50)$ & - & - & $15(31)$ & $17(27)$ \\
\hline Rheumatoid factor lgM, $n$ (\%) & $45(70)$ & - & - & $22(45)$ & $23(36)$ \\
\hline BASDAI & - & $4.73(0.3-9.5)$ & $5.1(0.9-9.6)$ & $5.5(0.3-9.3)$ & $5.1(0.9-9.7)$ \\
\hline BASFI & - & $3.2(0-9)$ & $4.1(1.1-7.6)$ & $3.0(0-9)$ & $3.8(0.4-8.6)$ \\
\hline BASMI & - & - & $3(0-10)$ & - & $3(0-10)$ \\
\hline DAS28 & $4.98(2.6-7.3)$ & - & - & $5.2(3.1-7.3)$ & $5.1(2.6-7.1)$ \\
\hline PtGA & $52(5-98)$ & $44(2-96)$ & $56(6-96)$ & $52(2-96)$ & $49(6-98)$ \\
\hline PGA & $38(7-73)$ & $21(0-57)^{¥}$ & $26(3-60)^{\phi}$ & $38(11-73)$ & $27(0-73)^{*}$ \\
\hline MHAQ & $0.65(0-1.45)$ & $0.40(0.05-1.55)$ & $0.43(0-1.40)$ & $0.45(1-1.55)$ & $0.50(0-1.40)$ \\
\hline \multicolumn{6}{|l|}{ Treatment, n (\%) } \\
\hline Anti-TNF monotherapy & $0(0)$ & $4(14)^{*}$ & $20(100)^{\phi \epsilon}$ & 0 & $24(38)^{*}$ \\
\hline MTX monotherapy & $34(53)$ & $15(52)$ & $0(0)^{\phi \epsilon}$ & $49(100)$ & $0(0)^{*}$ \\
\hline Anti-TNF \pm MTX & $30(47)$ & $10(34)$ & $0(0)^{\phi \epsilon}$ & $0(0)$ & $40(62)^{*}$ \\
\hline Beta-blockers & $5(8)$ & $1(3)$ & $4(20)$ & $4(8)$ & $6(9)$ \\
\hline Calcium antagonists & $5(8)$ & $2(7)$ & $2(10)$ & $2(4)$ & $7(11)$ \\
\hline ACE inhibitors & $6(9)$ & $1(3)$ & $2(10)$ & $4(8)$ & $5(8)$ \\
\hline NSAIDs & $47(73)$ & $14(48)^{*}$ & $14(70)$ & $35(71)$ & $40(62)$ \\
\hline Coxibs & $0(0)$ & $0(0)$ & $1(5)$ & $0(0)$ & $1(2)$ \\
\hline Statins & $12(19)$ & $1(3)^{\#}$ & $7(35)^{\epsilon}$ & $7(14)$ & $13(20)$ \\
\hline Acetyl salicylic acid & $6(9)$ & $2(7)$ & $3(15)$ & $6(12)$ & $5(8)$ \\
\hline Warfarin & $0(0)$ & $0(0)$ & $1(5)^{\epsilon}$ & $0(0)$ & $1(2)$ \\
\hline Glucocorticoids & $17(27)$ & $3(10)^{*}$ & $2(10)^{\phi}$ & $8(16)$ & $15(23)^{*}$ \\
\hline
\end{tabular}

Unless indicated otherwise, values are given as median (range)

Statistically significant differences are shown in bold typeface

${ }^{*} P<0.05$, versus MTX

${ }^{¥} P<0.05$, versus RA

$\epsilon_{P}<0.05$, versus PsA

${ }^{\oplus} P<0.05$, versus RA

ACE angiotensin converting enzyme, anti-TNF antitumor necrosis factor, AS ankylosing spondylitis, BASDAl Bath Ankylosing Spondylitis Disease Activity Index, BASFI Bath Ankylosing Spondylitis Functional Index, BASMI Bath Ankylosing Spondylitis Metrology Index, CRP C-reactive protein, DAS28 Disease activity score for 28 joints, ED endothelial dysfunction, ESR erythrocyte sedimentation rate, $l g$ immunoglobulin, MHAQ Medical Health Assessment Questionnaire, MTX methotrexate, NSAID nonsteroidal anti-inflammatory drug, NSJ number of swollen joints, PGA Physicians' Global Assessment Score of Disease Activity, PsA psoriatic arthritis, PtGA Patients' Global Assessment Score of Disease Activity, RA rheumatoid arthritis, RHI Reactive Hyperemic Index, WBC white blood cell

The RHI change from baseline to 6 months was not related to RHI baseline values in patients with ED.

RHI mean values in patients with normal EF did not change at any of the control points of time (data not shown).

\section{Discussion}

To our knowledge, this is the first study to compare the effect of MTX monotherapy and anti-TNF \pm MTX treatment on EF in IA patients, and to compare levels of RHI between RA, PsA, and AS patients with active disease.

In IA patients with $\mathrm{ED}$, antirheumatic treatment was associated with improvement in EF both at 6 weeks and 6 months of follow-up compared to baseline. However, after 6 weeks, EF continued to improve only in the MTX group. 
Table 2 Baseline cardiovascular characteristics for all patients

\begin{tabular}{|c|c|c|c|c|c|}
\hline & $\begin{array}{l}\text { RA } \\
(n=64)\end{array}$ & $\begin{array}{l}\text { PsA } \\
(n=29)\end{array}$ & $\begin{array}{l}\text { AS } \\
(n=20)\end{array}$ & $\begin{array}{l}\text { MTX } \\
(n=49)\end{array}$ & $\begin{array}{l}\text { anti-TNF } \pm \text { MTX } \\
(n=6)\end{array}$ \\
\hline \multicolumn{6}{|l|}{ Cardiovascular risk factors } \\
\hline Hypertension & $17(27)$ & $7(24)$ & $6(30)$ & $9(18)$ & $21(33)$ \\
\hline BMI $\left(\mathrm{kg} / \mathrm{m}^{2}\right)$, median (range) & $26(19-41)$ & $26(19-39)$ & $28(22-36)$ & $26(20-39)$ & $27(20-41)$ \\
\hline Hyperlipidemia & $11(17)$ & $3(10)$ & $3(15)$ & $9(18)$ & $8(12)$ \\
\hline Current smokers & $20(31)$ & $6(21)$ & $10(50)$ & $15(31)$ & $21(33)$ \\
\hline Family history of CVD or death & $33(52)$ & $13(45)$ & $10(50)$ & $24(50)$ & $32(50)$ \\
\hline Diabetes & $3(5)$ & $0(0)$ & $1(5)$ & $0(0)$ & $4(6)$ \\
\hline \multicolumn{6}{|l|}{ Medical history } \\
\hline Previous myocardial infarction & $5(8)$ & $0(0)$ & $2(10)$ & $2(4)$ & $5(8)$ \\
\hline Angina pectoris & $2(3)$ & $1(3)$ & $2(10)$ & $2(4)$ & $3(5)$ \\
\hline \multicolumn{6}{|l|}{ Endothelial dysfunction } \\
\hline RHI, median (range) & $1.89(1.24-2.94)$ & $2.06(1.45-2.94)$ & $1.81(1.37-2.72)^{\epsilon}$ & $1.93(1.24-2.76)$ & $1.82(1.37-2.94)$ \\
\hline ED & $22(34)$ & $9(31)$ & $9(45)$ & $18(37)$ & $22(34)$ \\
\hline RHI, median (range) for patients with ED & $1.47(1.24-1.65)$ & $1.56(1.45-1.64)$ & $1.52(1.37-1.64)$ & $1.49(1.24-1.63)$ & $1.52(1.37-1.65)$ \\
\hline
\end{tabular}

Unless indicated otherwise, values are given as number (percentage)

Statistically significant differences are shown in bold typeface

$\epsilon_{P<0.05}$, versus PsA

anti-TNF antitumor necrosis factor, $A S$ ankylosing spondylitis, $B M I$ body mass index, CVD cardiovascular disease, ED endothelial dysfunction, MTX methotrexate, Ps $A$ psoriatic arthritis, $R A$ rheumatoid arthritis, $R H I$ Reactive Hyperemic Index

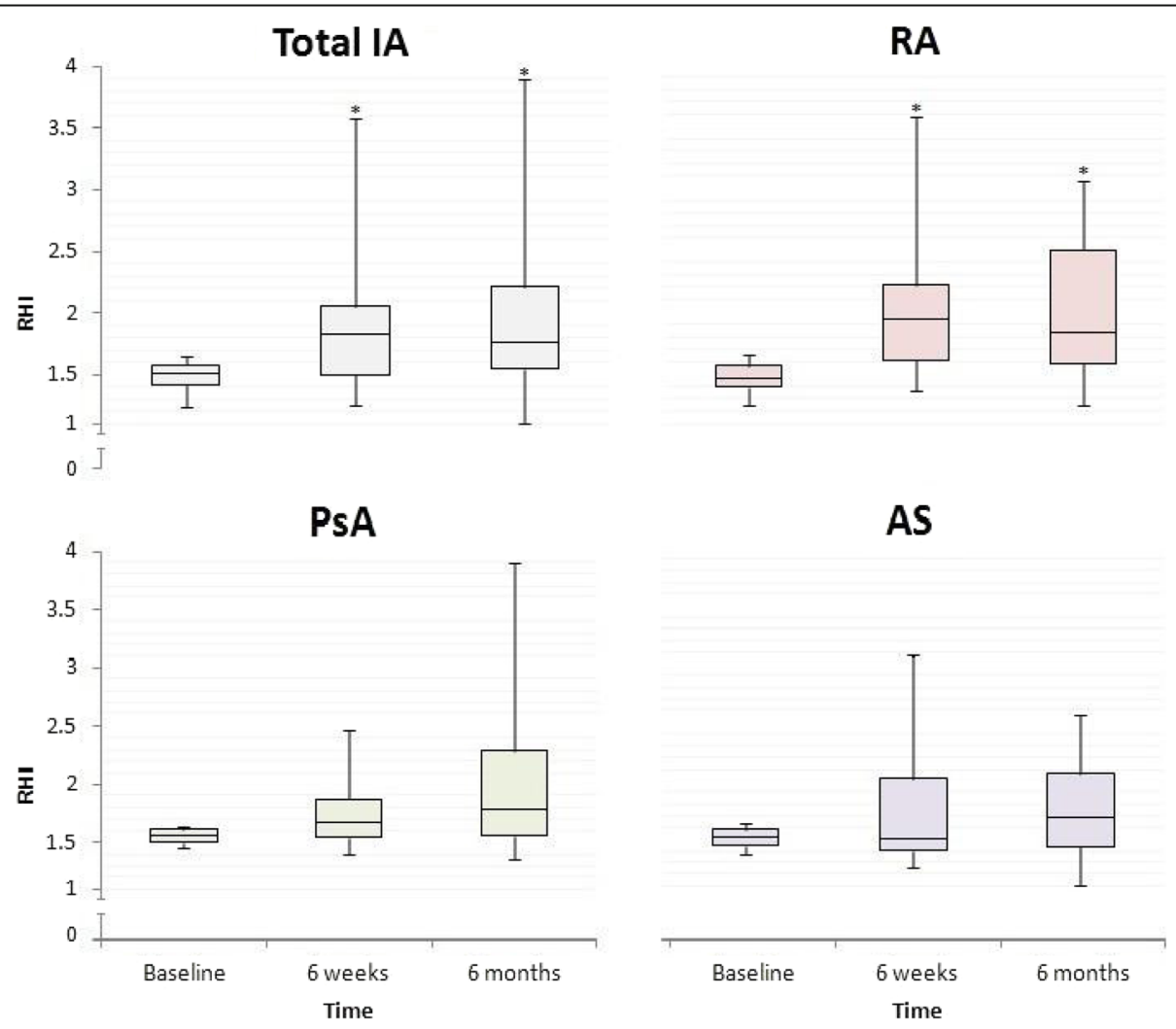

Fig. 1 RHI values in RA, PSA, and AS patients with ED at all visits. ${ }^{*} P<0.05$, versus baseline. The lines inside of the boxes show the median; the whiskers of the boxes show upper and lower values. AS ankylosing spondylitis, IA inflammatory arthritis, PsA psoriatic arthritis, RA rheumatoid arthritis, RHI Reactive Hyperemic Index 


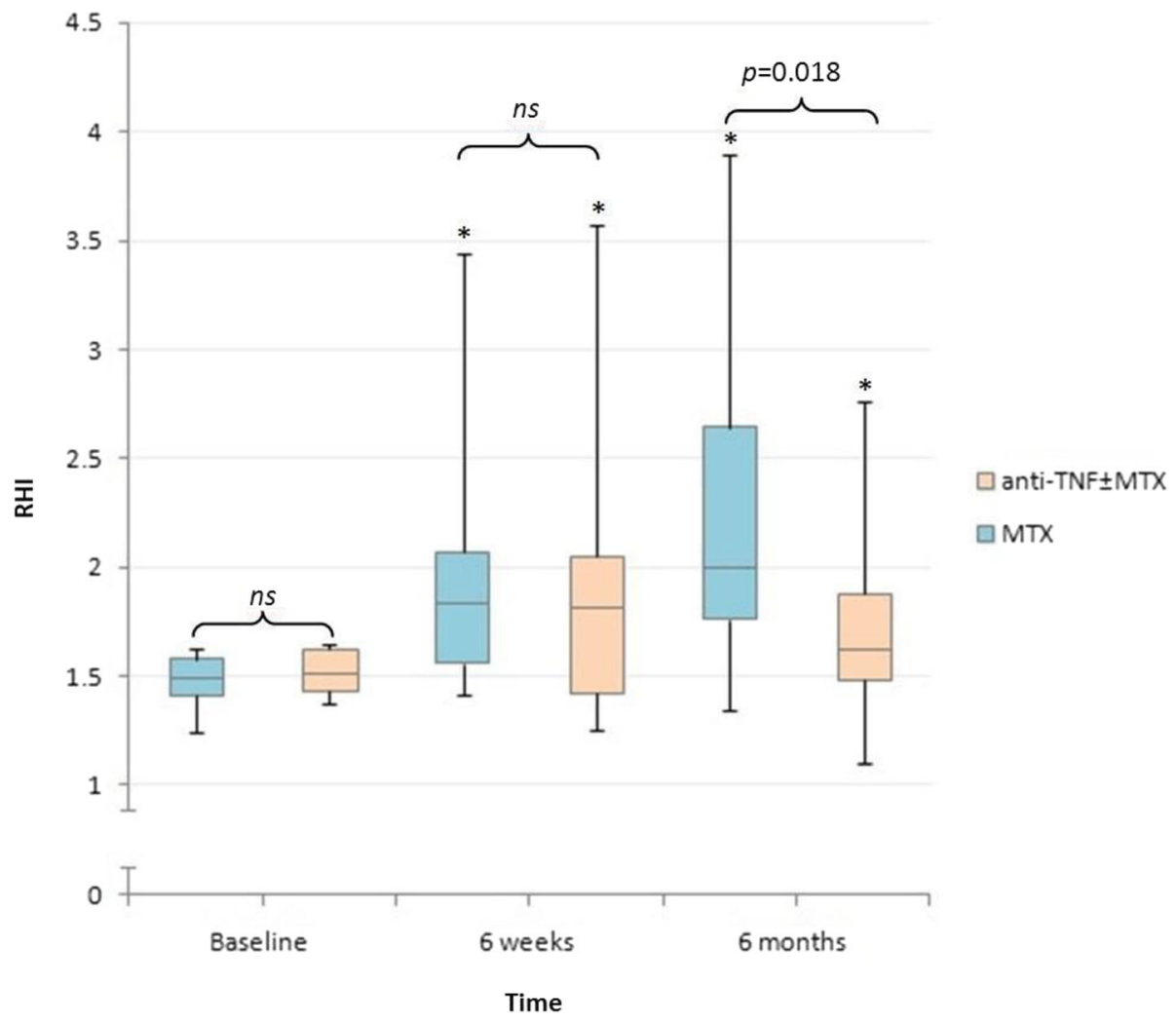

Fig. $2 \mathrm{RHI}$ values for patients with ED between and within the MTX and anti-TNF \pm MTX groups. ${ }^{*} P<0.05$ compared to baseline value. anti-TNF anti-tumor necrosis factor, MTX methotrexate, ns not statistically significant, RHI Reactive Hyperemic Index

Table 3 Predictors of RHI change after 6 months of antirheumatic treatment in patients with ED

\begin{tabular}{|c|c|c|c|c|c|c|}
\hline & \multicolumn{3}{|c|}{ Unadjusted } & \multicolumn{3}{|c|}{ Adjusted } \\
\hline & Beta & P & $95 \% \mathrm{Cl}$ & Beta & P & $95 \% \mathrm{Cl}$ \\
\hline Female gender & 0.492 & 0.011 & 0.118 to 0.865 & 0.621 & 0.004 & 0.220 to 1.022 \\
\hline Age & -0.004 & 0.669 & -0.024 to 0.016 & -0.001 & 0.919 & -0.021 to 0.019 \\
\hline Anti-TNF \pm MTX & -0.505 & 0.008 & -0.866 to -0.143 & -0.448 & 0.032 & -0.855 to -0.041 \\
\hline RDD & -0.026 & 0.033 & -0.049 to -0.002 & -0.024 & 0.068 & -0.050 to 0.002 \\
\hline PsA & -0.067 & 0.779 & -0.550 to 0.416 & 0.064 & 0.733 & -0.386 to 0.514 \\
\hline AS & -0.267 & 0.242 & -0.722 to 0.188 & 0.219 & 0.419 & -0.327 to 0.765 \\
\hline CRP & -0.004 & 0.523 & -0.017 to 0.009 & & & \\
\hline ESR & -0.002 & 0.771 & -0.017 to 0.013 & & & \\
\hline PTX3 & -0.071 & 0.255 & -0.194 to 0.053 & & & \\
\hline PGA & 0.008 & 0.160 & -0.003 to 0.019 & & & \\
\hline PtGA & -0.001 & 0.824 & -0.008 to 0.006 & & & \\
\hline MHAQ & -0.328 & 0.332 & -1.006 to 0.349 & & & \\
\hline NSJ & -0.150 & 0.050 & -0.299 to 0.000 & & & \\
\hline
\end{tabular}

Comparators: female gender versus male gender, anti-TNF \pm MTX versus MTX monotherapy

Statistically significant differences are shown in bold typeface

anti-TNF anti-tumor necrosis factor, AS ankylosing spondylitis, $C l$ confidence interval, CRP C-reactive protein, ESR erythrocyte sedimentation rate, MHAQ Medical Health Assessment Questionnaire, MTX methotrexate, NSJ number of swollen joints, PGA Physicians' Global Assessment Score of Disease Activity, PsA psoriatic arthritis, PtGA Patients' Global Assessment Score of Disease Activity, PTX3 pentraxin 3, RDD rheumatic disease duration, RHI Reactive Hyperemic Index 
Because MTX monotherapy was initiated only in MTX-naive patients, and the combination therapy only in patients who had previously used MTX without sufficient effect, our findings may indicate that MTX treatment in MTX-naive patients has a greater and more sustained vasculoprotective effect than anti-TNF monotherapy, or anti-TNF added to MTX treatment in MTX nonresponders. It is likely that, in MTX nonresponders, MTX also exhibited a poor response on disease activity after the addition of anti-TNF (MTX in this group was provided first of all to reduce side-effects of anti-TNF therapy). One might speculate that the poor response of MTX on inflammation is associated also with a poor effect on EF.

The exact mechanism behind the protective effect of antirheumatic treatment on ED is not known [25]. Theoretically, it might be mediated by inhibition of systemic inflammatory factors and the corresponding metabolic abnormalities. However, this explanation is not supported by our findings since the improvement in RHI was not related to systemic markers of disease activity, such as ESR and CRP. Moreover, we did not find any significant relationships between RHI levels and inflammatory markers at baseline.

Another explanation might be that the examined drugs might have a direct beneficial effect on the vessel walls, including the endothelium. It has been shown that MTX and anti-TNF treatments are associated with improvements in reverse cholesterol transport by various mechanisms [26, 27]. For example, MTX increases high-density lipoprotein (HDL) capacity to promote cholesterol efflux from cells [28]. Anti-TNF agents counteract the deleterious effects of TNF on the expression of genes involved in cholesterol efflux and reduce cell cholesterol accumulation through amelioration of serum lipoprotein functions and through reverse signaling following direct interaction with cell membrane-bound TNF [26].

Although most focus has been on the importance of impaired cell cholesterol efflux in the development of foam cells from macrophages in atherosclerotic plaques, the same mechanism may also underlie disturbances in endothelial cells, with reduction of their vasodilating and anti-inflammatory functions [29]. In fact, increased cholesterol efflux through the membrane transporters ATPbinding cassette A1 and G1 and Scavenger Receptor class B type I in endothelial cells is associated with promotion of eNOS expression and PGI2 production [30-32]. Moreover, serum HDL capacity to promote cell cholesterol efflux is directly correlated to flow mediated dilation [33]. Thus, the improved cell cholesterol efflux due to antirheumatic treatment might both protect from atheroma formation and from ED.

IA patients have been reported to have more inflammation, involving overexpression of TNF, in their vascular media and adventitia compared to non-IA patients with CVD [34, 35]. It might even be that inflammation located in deep vascular layers might affect the luminal part of the artery, including the phenotype of the endothelial cells [36]. Thus, in theory, antirheumatic treatment, such as anti-TNF, could also ameliorate EF by inhibition of vascular inflammation.

ED occurs when the endothelium is activated and is characterized by cytokine production, loss of vascular integrity, and expression of adhesion molecules [37]. Adhesion molecules such as intercellular adhesion molecule (ICAM)-1, E-selectin, and vascular cell adhesion molecule (VCAM)-1 make the endothelium surface more adhesive to leukocytes and facilitate their migration into the vessel wall (including atherosclerotic lesions) [37, 38].

In keeping with our results, both MTX and anti-TNF have been previously reported to downregulate expression of adhesion molecules on endothelial cells, i.e., circulating markers of ED [39-43]. Also, a recent review and meta-analysis article concluded that anti-TNF treatment might improve EF in RA patients [44].

Our previous article based on the same patient sample demonstrated that MTX and anti-TNF \pm MTX treatment significantly reduced inflammatory activity (determined by ESR, CRP, WBC count, PGA, and PtGA) both at 6 weeks and at 6 months compared to baseline [16]. This may indicate that both treatment regimens have a longstanding effect on inflammation, but only MTX (in patients potentially responding to it) has a prolonged beneficial effect on the endothelial cells.

We cannot definitely rule out the possibility that the observed differences in the effect of the antirheumatic treatments on EF might be based on differences in patient populations or other factors. For example, it might be that patients with longer and more therapy-resistant IA (i.e., features typical for the anti-TNF \pm MTX group; Table 1) had a higher CV risk and were less likely to improve their EF by antirheumatic treatment than the remaining IA patients (Table 2). Nevertheless, the differences in RHI change between baseline and 6 months in the two treatment groups were independent of rheumatic disease duration, IA diagnosis, and age. Moreover, there were no statistically significant differences in the examined traditional $\mathrm{CV}$ risk factors, the occurrences of clinical CVD and ED, and median RHI values at baseline between the two treatment groups (Table 2).

As different immune and other mechanisms are involved in the pathogenesis of RA, PsA, and AS, it might be that ED in these diseases is also mediated partly by different factors. Consequently, the effect of different antirheumatic drugs on ED in these particular diseases might also be different.

When comparing the three diagnostic groups, the AS group were the most likely to have ED and CV comorbidity 
(Table 2), in spite of being younger, having a similar disease duration as the RA and PsA groups, and being less likely to use systemic glucocorticoids than the RA group (Table 1). It is possible that the increased occurrence of certain $\mathrm{CV}$ risk factors, such as a high proportion of men and smokers, could partly explain the impaired EF in the AS group [45].

The improvements in RHI from baseline to 6 weeks and 6 months were apparent in all diagnostic groups, but it was statistically significant only in the total IA and in the RA group. The lack of statistically significant differences in RHI improvement in the other groups might be due to their relatively low sample size. Indeed, other studies indicate that antirheumatic treatment (anti-TNF) also improves EF in patients with PsA and AS [46, 47]. We cannot exclude the possibility that AS patients experienced less protection from antirheumatic treatment because they were treated only with anti-TNF and not MTX.

The cause of the observed decreased effect of anti-TNF on EF at 6 months is unclear. Among other factors, it might be caused by the well-known secondary nonresponse effect due to the development of antidrug antibodies [48].

Interestingly, women had statistically greater RHI improvement after 6 months of treatment than men (Table 2). Thus, our results may indicate that women have a better ability to reverse ED than men, independently of IA diagnosis, when treated with MTX or anti-TNF \pm MTX. We do not know the molecular mechanism behind this phenomenon.

Rheumatic disease duration showed a stable negative association with RHI change from baseline to 6 months in several multiple regression models. It seems more difficult to achieve an EF improvement in patients with longer rheumatic disease duration, and this applies for both treatment regimens. Thus, these data support the notion that early antirheumatic treatment is important not only for prevention of joint damage, but also for protection from atherosclerosis. However, our results have to be confirmed in larger studies.

As in most studies, ours has several limitations. First, due to ethical reasons (to avoid prescribing MTX to patients in need of anti-TNF, and to avoid overtreatment in patients that might be sufficiently treated with MTX monotherapy) we conducted an observational study instead of a randomized controlled trial (RCT). Thus, we could not secure the same level of similarity between study groups at baseline as in a RCT, nor conduct double-blinded evaluation. On the other hand, observational studies have other advantages, e.g., they can more accurately reflect real life, and therefore have increasingly been called for over the last years. To compensate for baseline differences between the groups, we adjusted for several baseline characteristics in multiple regression models.
As MTX is the drug of choice in most patients with peripheral chronic arthritis, patients with these conditions who receive anti-TNF treatment are likely to have a longer and more severe IA. Nevertheless, the anti-TNF group did not differ from the MTX group when comparing several disease activity markers. In fact, the MTX group had statistically significantly higher PGA scores than the anti-TNF \pm MTX group (Table 1).

Second, we were not able to evaluate differences in monotherapies with MTX and anti-TNF as most of the patients using anti-TNF also used MTX comedication.

Third, we evaluated RHI change only in patients with ED because we could not expect substantial RHI improvement in patients with normal EF. Therefore, regression to the mean might be questioned. However, in contrast to patients with low RHI, RHI mean values in patients with normal EF did not change towards the RHI mean at any of the control points of time. Taken together, these observations diminish the suspicion that the observed RHI differences in the ED group could be explained by regression to the mean only.

Finally, owing to a relatively small sample size, the apparent lack of some differences and associations may be due to type II errors and insufficient statistical power. Still, as this is to our knowledge the first study comparing the effect of MTX and anti-TNF regimens in IAs on EF, and comparing EF in RA, PsA, and AS, it brings new important insights into CVD in IAs, and indicates the need for further research.

An advantage of our study is a well-characterized study sample, and a design that makes it possible to examine the effect of two of the main antirheumatic treatment regimens on EF in three common IAs.

\section{Conclusions}

In conclusion, treatment with MTX and anti-TNF \pm MTX appears to improve EF relatively quickly in IA patients with ED. After 6 months, the EF improvement was more pronounced in the MTX users than in the anti-TNF \pm MTX users. Among other factors, this might be due to a more sustained beneficial effect of MTX on the vasculature.

Because the EF improvement was independent of improvement in rheumatic disease activity, modes of action other than the anti-inflammatory effect might play a role.

Among patients with active RA, AS, and PsA, those with AS had the worst endothelial function (the difference was statistically significantly different compared to those with PsA), although they were the youngest.

\footnotetext{
Abbreviations

AS: Ankylosing spondylitis; CRP: C-reactive protein; CV: Cardiovascular;

CVD: Cardiovascular disease; DMARD: Disease-modifying antirheumatic drug; ED: Endothelial dysfunction; EF: Endothelial function; ESR: Erythrocyte

sedimentation rate; HDL: High-density lipoprotein; IA: Inflammatory arthritis; ICAM: intercellular adhesion molecule; MHAQ: Modified Health Assessment Questionnaire; MTX: Methotrexate; NSAID: Nonsteroidal anti-inflammatory
} 
drug; PGA: Physicians' Global Assessment Score of Disease Activity; PsA: Psoriatic arthritis; PSARA: PSoriatic arthritis, Ankylosing spondylitis, Rheumatoid Arthritis; PtGA: Patients' Global Assessment Score of Disease Activity; PTX: Pentraxin; PWA: Pulse wave amplitude; RA: Rheumatoid arthritis; RCT: Randomized controlled trial; RHI: Reactive Hyperemic Index; RHPAT: Reactive hyperemia peripheral arterial tonometry; SpA: Spondyloarthritis; TNF: Tumor necrosis factor; VCAM: Vascular cell adhesion molecule: WBC: White blood cel

\section{Acknowledgements}

We are grateful to the whole PSARA network involved in the establishment and implementation of the PSARA study, in particular to Allan Wiik (Statens Serum Institute, Copenhagen, Denmark), Øystein T. Førre (University of Oslo, Norway), and Bente Malerbakken and Chevy Stubberud (Lillehammer Hospital of Rheumatic Diseases, Lillehammer, Norway).

\section{Funding}

This study has been supported by a grant from Innlandet Hospital Trust. Abbot Norway financed the establishment of the PSARA biobank, but had no direct involvement in the study described in this article (including design, data collection and analysis, and manuscript preparation).

\section{Availability of data and materials}

All the supporting data are available upon request.

\section{Authors' contributions}

IHok contributed with acquisition of data and drafting the manuscript. $\mathrm{Hol}_{\text {, }}$ $T L, S A$, and GH were the founders of the PSARA study and contributed to conception and design, and analysis and interpretation of data. IHol was also involved in drafting the manuscript. MCS and GD performed the statistical analysis and interpreted the results. GD was the major contributor to writing the manuscript. KM, NR, and JEW contributed to interpretation of data. All authors have been involved in critically revising the manuscript. All authors read and approved the final manuscript and agreed to be accountable for all aspects of the work.

\section{Authors' information}

Not applicable

\section{Ethics approval and consent to participate}

The Norwegian Regional Ethical Committee approved the study protocol and all patients gave informed written consent.

\section{Consent for publication}

All patients gave informed written consent to publish.

\section{Competing interests}

The authors declare that they have no competing interests.

\section{Publisher's Note}

Springer Nature remains neutral with regard to jurisdictional claims in published maps and institutional affiliations.

\section{Author details}

'Department of Medical Biochemistry, Innlandet Hospital Trust, Hamar, Norway. 'Lillehammer Hospital for Rheumatic Diseases, Lillehammer, Norway. ${ }^{3}$ Institution of Health Care, Health Science PhD Program, Oslo and Akershus University College, Oslo, Norway. ${ }^{4}$ Oslo University Hospital, Ullevål, Oslo, Norway. ${ }^{5}$ Institute of Clinical Sciences, University of Oslo, Oslo, Norway. ${ }^{6}$ Department of Medical Biochemistry, Oslo University Hospital, Ullevål, Oslo, Norway. ${ }^{7}$ Department of Food and Drug, University of Parma, Parma, Italy. ${ }^{8}$ Department of Medicine, Innlandet Hospital Trust, Lillehammer, Norway. ${ }^{9}$ Department of Medicine, Brigham and Women's Hospital, Boston, USA ${ }^{10}$ Harvard Medical School, Boston, USA. ${ }^{11}$ Department of Research, Innlandet Hospital Trust, Brumunddal, Norway.
}

Received: 23 May 2017 Accepted: 20 September 2017

Published online: 17 October 2017

\section{References}

1. Hollan I, Meroni PL, Ahearn JM, Cohen Tervaert JW, Curran S, Goodyear CS, et al. Cardiovascular disease in autoimmune rheumatic diseases. Autoimmun Rev. 2013:12(10):1004-15.

2. Boehncke WH, Boehncke S. Cardiovascular mortality in psoriasis and psoriatic arthritis: epidemiology, pathomechanisms, therapeutic implications, and perspectives. Curr Rheumatol Rep. 2012;14(4):343-8.

3. Gladman DD, Farewell VT, Wong K, Husted J. Mortality studies in psoriatic arthritis: results from a single outpatient center. II. Prognostic indicators for death. Arthritis Rheum. 1998:41(6):1103-10.

4. Lehtinen K. Mortality and causes of death in 398 patients admitted to hospital with ankylosing spondylitis. Ann Rheum Dis. 1993;52(3):174-6.

5. Han C, Robinson DW, Hackett MV, Paramore LC, Fraeman KH, Bala MV. Cardiovascular disease and risk factors in patients with rheumatoid arthritis, psoriatic arthritis, and ankylosing spondylitis. J Rheumatol. 2006;33(11):2167-72.

6. Celermajer DS. Endothelial dysfunction: does it matter? Is it reversible? J Am Coll Cardiol. 1997:30(2):325-33.

7. Behrendt D, Ganz P. Endothelial function: from vascular biology to clinical applications. Am J Cardiol. 2002;90(10, Supplement 3):L40-8.

8. Gonzalez-Juanatey C, Llorca J, Miranda-Filloy JA, Amigo-Diaz E, Testa A, Garcia-Porrua C, et al. Endothelial dysfunction in psoriatic arthritis patients without clinically evident cardiovascular disease or classic atherosclerosis risk factors. Arthritis Rheum. 2007:57(2):287-93.

9. Nadar S, Blann AD, Lip GY. Endothelial dysfunction: methods of assessment and application to hypertension. Curr Pharm Des. 2004;10(29):3591-605.

10. Sari I, Okan T, Akar S, Cece H, Altay C, Secil M, et al. Impaired endothelial function in patients with ankylosing spondylitis. Rheumatology (Oxford). 2006;45(3):283-6.

11. Bonetti PO, Lerman LO, Lerman A. Endothelial dysfunction: a marker of atherosclerotic risk. Arterioscler Thromb Vasc Biol. 2003;23(2):168-75.

12. Barnabe C, Martin BJ, Ghali WA. Systematic review and meta-analysis: antitumor necrosis factor alpha therapy and cardiovascular events in rheumatoid arthritis. Arthritis Care Res (Hoboken). 2011;63(4):522-9.

13. Westlake SL, Colebatch AN, Baird J, Kiely P, Quinn M, Choy E, et al. The effect of methotrexate on cardiovascular disease in patients with rheumatoid arthritis: a systematic literature review. Rheumatology (Oxford). 2010;49(2):295-307.

14. Hürlimann D, Forster A, Noll G, Enseleit F, Chenevard R, Distler O, Béchir M, Spieker LE, Neidhart M, Michel BA, Gay RE, Lüscher TF, Gay S, Ruschitzka F, et al. Anti-tumor necrosis factor treatment improves endothelial function in patients with rheumatoid arthritis. Circulation. 2002;106:2184-7.

15. Angel K, Provan SA, Fagerhol MK, Mowinckel P, Kvien TK, Atar D. Effect of 1year anti-TNF-a therapy on aortic stiffness, carotid atherosclerosis, and calprotectin in inflammatory arthropathies: a controlled study. Am J Hypertens. 2012;25(6):644-50.

16. Deyab G, Hokstad I, Whist JE, Smastuen MC, Agewall S, Lyberg T, et al. Antirheumatic treatment is not associated with reduction of pentraxin 3 in rheumatoid arthritis, psoriatic arthritis and ankylosing spondylitis. PLoS One. 2017:12(2):e0169830

17. Moll JMH, Wright V. Psoriatic arthritis. Semin Arthritis Rheum. 1973;3(1):55-78

18. van der Linden S, Valkenburg HA, Cats A. Evaluation of diagnostic criteria for ankylosing spondylitis. A proposal for modification of the New York criteria. Arthritis Rheum. 1984:27(4):361-8.

19. Arnett FC, Edworthy SM, Bloch DA, McShane DJ, Fries JF, Cooper NS, et al. The American Rheumatism Association 1987 revised criteria for the classification of rheumatoid arthritis. Arthritis Rheum. 1988;31(3):315-24.

20. Pavy S, Constantin A, Pham T, Gossec L, Maillefert J-F, Cantagrel A, et al. Methotrexate therapy for rheumatoid arthritis: clinical practice guidelines based on published evidence and expert opinion. Joint Bone Spine. 2006;73(4):388-95.

21. Ward MM, Deodhar A, Akl EA, Lui A, Ermann J, Gensler LS, et al. American College of Rheumatology/Spondylitis Association of America/ Spondyloarthritis Research and Treatment Network 2015 recommendations for the treatment of ankylosing spondylitis and nonradiographic axial spondyloarthritis. Arthritis Rheumatol. 2016;68(2):282-98.

22. van der Heijde D, Sieper J, Maksymowych WP, Dougados M, Burgos-Vargas $\mathrm{R}$, Landewe $\mathrm{R}$, et al. 2010 Update of the international ASAS 
recommendations for the use of anti-TNF agents in patients with axia spondyloarthritis. Ann Rheum Dis. 2011;70(6):905-8.

23. Bonetti PO, Barsness GW, Keelan PC, Schnell TI, Pumper GM, Kuvin JT, et al. Enhanced external counterpulsation improves endothelial function in patients with symptomatic coronary artery disease. J Am Coll Cardiol. 2003:41(10):1761-8.

24. Hjeltnes G, Hollan I, Førre Ø, Wiik A, Lyberg T, Mikkelsen K, Agewall S. Endothelial function improves within 6 weeks of treatment with methotrexate or methotrexate in combinition with a TNF - a inhibitor in rheumatoid arthritis patients. Scand J Rheumatol. 2012;41:240-8

25. Yang $X$, Chang $Y$, Wei W. Endothelial dysfunction and inflammation: immunity in rheumatoid arthritis. Mediators Inflamm. 2016;2016:6813016.

26. Voloshyna I, Seshadri S, Anwar K, Littlefield MJ, Belilos E, Carsons SE, et al. Infliximab reverses suppression of cholesterol efflux proteins by TNF-a: a possible mechanism for modulation of atherogenesis. Biomed Res Int. 2014;2014:8

27. Reiss AB, Carsons SE, Anwar K, Rao S, Edelman SD, Zhang H, et al. Atheroprotective effects of methotrexate on reverse cholesterol transport proteins and foam cell transformation in human THP-1 monocyte/ macrophages. Arthritis Rheum. 2008;58(12):3675-83.

28. Ronda N, Greco D, Adorni MP, Zimetti F, Favari E, Hjeltnes G, et al. Newly identified antiatherosclerotic activity of methotrexate and adalimumab: complementary effects on lipoprotein function and macrophage cholesterol metabolism. Arthritis Rheumatol. 2015;67(5):1155-64.

29. Prosser HC, Ng MK, Bursill CA. The role of cholesterol efflux in mechanisms of endothelial protection by HDL. Curr Opin Lipidol. 2012;23(3):182-9.

30. Terasaka N, Yu S, Yvan-Charvet L, Wang N, Mzhavia N, Langlois R, et al. $A B C G 1$ and $H D L$ protect against endothelial dysfunction in mice fed a highcholesterol diet. J Clin Invest. 2008;1 18(11):3701-13.

31. Terasaka N, Westerterp M, Koetsveld J, Fernandez-Hernando C, Yvan-Charvet L, Wang N, et al. ATP-binding cassette transporter G1 and high-density lipoprotein promote endothelial NO synthesis through a decrease in the interaction of caveolin-1 and endothelial NO synthase. Arterioscler Thromb Vasc Biol. 2010;30(11):2219-25.

32. Liu D, Ji L, Tong X, Pan B, Han JY, Huang Y, et al. Human apolipoprotein A-I induces cyclooxygenase-2 expression and prostaglandin 1-2 release in endothelial cells through ATP-binding cassette transporter A1. Am J Physiol Cell Physiol. 2011;301(3):C739-48.

33. Vigna GB, Satta E, Bernini F, Boarini S, Bosi C, Giusto L, et al. Flow-mediated dilation, carotid wall thickness and HDL function in subjects with hyperalphalipoproteinemia. Nutr Metab Cardiovasc Dis. 2014;24(7):777-83.

34. Ahmed A, Hollan I, Curran SA, Kitson SM, Riggio MP, Mikkelsen K, et al. Brief report: proatherogenic cytokine microenvironment in the aortic adventitia of patients with rheumatoid arthritis. Arthritis Rheumatol. 2016;68(6):1361-6.

35. Shelef MA, Sokolove J, Lahey $\amalg$, Wagner CA, Sackmann EK, Warner TF, et al. Peptidylarginine deiminase 4 contributes to tumor necrosis factor alphainduced inflammatory arthritis. Arthritis Rheumatol. 2014;66(6):1482-91.

36. Savoia C, Sada L, Zezza L, Pucci L, Lauri FM, Befani A, et al. Vascular inflammation and endothelial dysfunction in experimental hypertension. Int J Hypertens. 2011;2011:281240.

37. Hunt BJ, Jurd KM. Endothelial cell activation: a central pathophysiological process. BMJ. 1998;316(7141):1328-9.

38. Deanfield JE, Halcox JP, Rabelink TJ. Endothelial function and dysfunction: testing and clinical relevance. Circulation. 2007;115(10):1285-95.

39. Johnston A, Gudjonsson JE, Sigmundsdottir H, Ludviksson BR, Valdimarsson $H$. The anti-inflammatory action of methotrexate is not mediated by lymphocyte apoptosis, but by the suppression of activation and adhesion molecules. Clin Immunol. 2005;114(2):154-63.

40. Silva LC, Ortigosa LC, Benard G. Anti-TNF-alpha agents in the treatment of immune-mediated inflammatory diseases: mechanisms of action and pitfalls. Immunotherapy. 2010;2(6):817-33.

41. Klimiuk PA, Sierakowski S, Domyslawska I, Fiedorczyk M, Chwiecko J. Reduction of soluble adhesion molecules (SICAM-1, SVCAM-1, and SEselectin) and vascular endothelial growth factor levels in serum of rheumatoid arthritis patients following multiple intravenous infusions of infliximab. Arch Immunol Ther Exp (Warsz). 2004;52(1):36-42.

42. Hjeltnes G, Hollan I, Forre O, Wiik A, Lyberg T, Mikkelsen K, et al. Serum levels of lipoprotein(a) and E-selectin are reduced in rheumatoid arthritis patients treated with methotrexate or methotrexate in combination with TNF-alpha-inhibitor. Clin Exp Rheumatol. 2013;31(3):415-21.
43. Huang Z, Chen C, Li S, Kong F, Shan P, Huang W. Serum markers of endothelial dysfunction and inflammation increase in hypertension with prediabetes mellitus. Genet Test Mol Biomarkers. 2016;20(6):322-7.

44. Ursini F, Leporini C, Bene F, D'Angelo S, Mauro D, Russo E, et al. Anti-TNFalpha agents and endothelial function in rheumatoid arthritis: a systematic review and meta-analysis. Sci Rep. 2017;7(1):5346.

45. Hollan I, Scott H, Saatvedt K, Prayson R, Mikkelsen K, Nossent HC, et al. Inflammatory rheumatic disease and smoking are predictors of aortic inflammation: a controlled study of biopsy specimens obtained at coronary artery surgery. Arthritis Rheum. 2007;56(6):2072-9.

46. van Eijk IC, Peters MJ, Serne EH, van der Horst-Bruinsma IE, Dijkmans BA, Smulders $Y M$, et al. Microvascular function is impaired in ankylosing spondylitis and improves after tumour necrosis factor alpha blockade. Ann Rheum Dis. 2009;68(3):362-6.

47. Brezinski EA, Follansbee MR, Armstrong EJ, Armstrong AW. Endothelial dysfunction and the effects of TNF inhibitors on the endothelium in psoriasis and psoriatic arthritis: a systematic review. Curr Pharm Des. 2014;20(4):513-28

48. Jani $M$, Barton $A$, Warren $R B$, Griffiths $C E$, Chinoy $H$. The role of DMARDs in reducing the immunogenicity of TNF inhibitors in chronic inflammatory diseases. Rheumatology (Oxford). 2014;53(2):213-22.

\section{Submit your next manuscript to BioMed Central and we will help you at every step:}

- We accept pre-submission inquiries

- Our selector tool helps you to find the most relevant journal

- We provide round the clock customer support

- Convenient online submission

- Thorough peer review

- Inclusion in PubMed and all major indexing services

- Maximum visibility for your research

Submit your manuscript at www.biomedcentral.com/submit
Biomed Central 